\title{
Philosophiques
}

\section{Le langage comme calcul dans le Big Typescript}

\section{Mauro Engelmann et Bento Prado Neto}

Volume 39, numéro 1, printemps 2012

La période intermédiaire de Wittgenstein

URI : https://id.erudit.org/iderudit/1011609ar

DOI : https://doi.org/10.7202/1011609ar

Aller au sommaire du numéro

Éditeur(s)

Société de philosophie du Québec

ISSN

0316-2923 (imprimé)

1492-1391 (numérique)

Découvrir la revue

Citer cet article

Engelmann, M. \& Prado Neto, B. (2012). Le langage comme calcul dans le Big Typescript. Philosophiques, 39(1), 35-55. https://doi.org/10.7202/1011609ar

\section{Résumé de l'article}

Dans cet article, j'essaie de montrer que l'idée du langage comme calcul structure la philosophie de Wittgenstein dans le Big Typescript. Pour ce faire, je commence par mettre en relief les différences entre la conception du langage comme calcul dans le Tractatus, et sa reformulation dans le Big Typescript ; j'explique ensuite comment l'idée de l'autonomie de la grammaire est à la base de la conception de la " grammaire » ou du langage comme calcul. J'espère pouvoir montrer ainsi l'unité de la philosophie de Wittgenstein aux alentours de 1932-1933. Il doit en ressortir que sa philosophie à l'époque du Big Typescript présente un intérêt en elle-même, quoiqu'il ne s'agisse pas encore de la philosophie des Recherches philosophiques.
Ce document est protégé par la loi sur le droit d'auteur. L'utilisation des services d'Érudit (y compris la reproduction) est assujettie à sa politique d'utilisation que vous pouvez consulter en ligne.

https://apropos.erudit.org/fr/usagers/politique-dutilisation/ 


\title{
Le langage comme calcul dans le Big Typescript
}

\author{
MAURO ENGELMANN \\ Université fédérale de Minas Gerais, Brésil \\ mauroengelmann@gmail.com
}

\begin{abstract}
RÉSUMÉ. - Dans cet article, j'essaie de montrer que l'idée du langage comme calcul structure la philosophie de Wittgenstein dans le Big Typescript. Pour ce faire, je commence par mettre en relief les différences entre la conception du langage comme calcul dans le Tractatus, et sa reformulation dans le Big Typescript; j'explique ensuite comment l'idée de l'autonomie de la grammaire est à la base de la conception de la «grammaire» ou du langage comme calcul. J'espère pouvoir montrer ainsi l'unité de la philosophie de Wittgenstein aux alentours de 1932-1933. Il doit en ressortir que sa philosophie à l'époque du Big Typescript présente un intérêt en elle-même, quoiqu'il ne s'agisse pas encore de la philosophie des Recherches philosophiques.
\end{abstract}

\begin{abstract}
I aim at showing that the idea of language as a calculus is the central view that shapes Wittgenstein's philosophy in the Big Typescript. In order to do so, I first contrast the conception of language as a calculus in the Tractatus with its reformulation in the Big Typescript; then I explain how the idea of the autonomy of grammar grounds the conception of "grammar" or language as calculus. With this on hand, I give an overview of the unity of Wittgenstein's philosophy around 1932-1933. It should become clear that his philosophy at the time is worth arguing for, even though it is not yet the philosophy of the Philosophical Investigations.
\end{abstract}

\section{Introduction}

Dans la première partie de cet article, j'avance que Wittgenstein conçoit la grammaire dans le Big Typescript (dorénavant cité BT) comme un calcul à règles fixes qui établit ce qui est et ce qui n'est pas permis dans le langage ${ }^{1}$. En cela, le Big Typescript et le Tractatus sont très proches l'un de l'autre. Cependant, la conception de la nature absolument a priori de ce calcul qui était défendue dans le Tractatus ne l'est plus dans le Big Typescript ${ }^{2}$. Cela

1. J'ai employé les trois versions disponibles du Big Typescript (dorénavant BT). L'exemplaire avec des modifications et des remarques manuscrites (Wittgenstein, 2005), «l'exemplaire vierge» de la Wiener Ausgabe (Wittgenstein, 20oob) et l'exemplaire électronique de la Bergen Edition (les références renvoient aux numéros de pages $d u$ tapuscrit; les traductions des textes du BT s'appuient, autant que possible, sur la traduction française de la Grammaire philosophique; pour les autres textes de Wittgenstein, sauf mention, les traductions sont celles du traducteur.)

2. Il faut avertir le lecteur que je n'ai pas l'intention de traiter ici de la méthode génétique utilisée par Wittgenstein dans le Big Typescript. Celle-ci consiste en une investigation de la genèse des puzzles philosophiques (en un repérage des fausses analogies qui les produisent) et en l'expression claire des trains de pensée trompeurs qui sont à la base de leur formulation. Je me limiterai ici à exposer la conception du langage comme calcul, une conception qui fonde et guide l'application de la méthode dans le Big Typescript. 
mène Wittgenstein à une conception de la nécessité de la règle qui la rend toujours relative à un système ${ }^{3}$. La conception du langage comme calcul dans le Big Typescript peut donc être vue comme une reformulation ou une adaptation significative de la philosophie du Tractatus. Dans la deuxième partie, j'explique brièvement l'autonomie du calcul. J'éclaircis l'autonomie du calcul en indiquant comment la réalité «se lie » aux propositions et aux attentes. Dans la troisième partie, j'explique comment la conception du langage comme calcul vise à déjouer la psychologisation de la "grammaire» et sert d'outil pour mettre en corrélation divers concepts mentaux ( "penser», "vouloir dire », "comprendre ", etc.) à l'intérieur d'un système. Finalement, j'essaie de mettre en lumière les opérations du calcul en indiquant comment cette idée est utilisée pour prouver que certaines questions philosophiques sont dépourvues de sens. L'idée fondamentale des trois parties de cet article est que la conception du langage comme calcul fait ressortir l'unité de la philosophie de Wittgenstein à l'époque du Big Typescript $t^{4}$. Je suggère également (sans m'y attarder) que la reformulation de la philosophie de Wittgenstein dans le Big Typescript ne doit pas être identifiée à la philosophie des Recherches philosophiques ${ }^{5}$.

3. La position de Wittgenstein dans le Big Typescript est une solution de rechange intéressante (avant la lettre) dans le débat Carnap-Quine. Je ne peux pas m'y attarder ici (voir, cependant, la note I6).

4. Kienzler $(2006$, I 8$)$ a sommé les interprètes de montrer que le Big Typescript est une œuvre ciblée. C'est précisément ce que j'ai l'intention de faire ici. Dans un certain sens, l'unité du Big Typescript (TS 2 $\mathrm{I}_{3}$ ) est déjà présente dans les Philosophische Bemerkungen (TS209) de I930: dans les deux compilations de remarques, Wittgenstein indique que son but est de composer un livre de "grammaire ", un livre dans lequel des règles "grammaticales» et des "genres de mots" liés aux fausses conceptions philosophiques au sujet de la phénoménologie, de la logique, et des mathématiques sont présentés afin de nettoyer le langage (voir BT I I 5 et Philosophische Bemerkungen $\$ 3$ ). Cela explique à mon avis les divisions et les titres du Big Typescript. Ce qui distingue les deux tapuscrits est précisément la centralité que le Big Typescript accorde à l'idée du langage comme calcul (ou jeu) et l'utilisation de cette idée afin d'éclaircir les concepts psychologiques. Les manuscrits (MSS) et les tapuscrits (TSS) sont numérotés selon le catalogue de Von Wright (I980) et sont cités d'après la Wiener Ausgabe.

5. Je ne m'occuperai pas dans cet article d'un aspect central de la philosophie de Wittgenstein dans le Big Typescript, à savoir l'emploi de la méthode génétique. La méthode peut être caractérisée de la façon suivante: beaucoup de questions philosophiques trouvent leur origine dans des analogies et des images trompeuses et des faux trains de pensées; le but de Wittgenstein est de montrer comment et pourquoi de tels problèmes surgissent; une fois qu'on l'a fait, les analogies et les images trompeuses, ainsi que les faux trains de pensées sont présentés au lecteur sous forme de miroir, de telle sorte qu'il puisse les reconnaître comme étant les siens (sur l'invention d'une nouvelle méthode, voir Engelmann, 20 I 2 b). À mon avis, la conception du langage comme calcul, telle qu'elle est présentée ici, est à la base de l'application de cette méthode dans le Big Typescript. Ce n'est cependant pas le cas dans les derniers textes de Wittgenstein. En ce qui concerne l'évolution de la philosophie de Wittgenstein, voir Engelmann (20I2a). 


\section{Le calcul, les limites du sens, et le Tractatus}

Dans le Big Typescript, le langage, le calcul et le système de règles sont considérés comme équivalents entre eux: "Ce qui vaut pour le mot "langage" doit également valoir pour l'expression "système de règles". Et donc aussi pour le mot "calcul” » (BT 65).

Cette équivalence implique que le langage fonctionne comme un calcul déterminé par des règles. Wittgenstein l'exprime également par la comparaison entre le langage et le jeu: "Le langage ne fonctionne comme un langage que grâce aux règles que nous observons en l'utilisant, comme un jeu ne fonctionne comme jeu que grâce à des règles ${ }^{6}$ "(BT I96 et I98; c'est moi qui souligne). L'idée de la grammaire et du langage en tant que "calcul» (ou que «jeu») est ainsi fondée sur l'idée que les opérations du langage (le fonctionnement de toutes les sortes d'énoncés) sont déterminées par des règles fixes:

Je considère le langage et la grammaire du point de vue d'un calcul// sous la forme d'un calcul// comme un calcul, c.-à-d., fonctionnant selon des règles fixes// comme un processus qui suit des règles fixes ${ }^{7}$ [BT 258 ; mises en relief et variantes dans le texte].

Les «règles fixes» du "calcul» dans le Big Typescript sont censées nous indiquer "ce qui peut compter comme une proposition" (BT 76), c.-à-d. quelles combinaisons de mots veulent dire quelque chose et lesquelles sont un non-sens: "Les règles grammaticales déterminent le sens d'une proposition; et aussi, si une combinaison de mots est pourvue de sens» (BT, 79). Comme dans le Tractatus, la signification des mots est dépendante d'un contexte ou d'un système: qu'une combinaison de mots soit pourvue de sens, c'est ce qui n'est pas déterminé par la signification prétendument isolée des mots, mais seulement par les règles qui constituent la signification dans le système (BT 3). C'est le sens des énoncés complets (leur rôle) dans le système du calcul du langage qui détermine quelles combinaisons de mots sont pourvues de $\operatorname{sens}^{8}$ (BT: I, 8I).

Les «limites du sens» sont donc établies par les règles grammaticales qui décrivent et la structure du langage et ce qu'il permet (ses possibilités): "On peut dire que les règles grammaticales décrivent la structure du langage, décrivent ses possibilités ${ }^{9} »$ (BT I95, note marginale). La détermination de cette structure englobe toutes les conditions de sens:

6. Et aussi: «La logique aristotélicienne // le syllogisme // est un jeu qui // est un calcul qui // peut être appliqué aux propositions» (BT 260).

7. Ce même point de vue est exprimé en plusieurs passages (voyez, par exemple, BT 65, $8 \mathrm{I}, \mathrm{IOO}, \mathrm{II} 2, \mathrm{I} 43)$.

8. Le rapport entre le calcul et la proposition est rendu explicite par Wittgenstein dans BT I43: «Nous ne nous intéressons qu'au contenu d'une proposition [...]. Une proposition a son contenu en tant que partie d'un calcul» (BT I43).

9. Pour ce qui concerne la grammaire et le sens, Wittgenstein dit dans ses Lent lectures de I93 I : «La grammaire (règles et vocabulaire) est la description du langage, et elle consiste 
La seule chose qui n'appartient pas à la grammaire est ce qui constitue la vérité et la fausseté d'une proposition. C'est la seule chose qui ne concerne pas la grammaire. Toutes les conditions pour une comparaison de l'énoncé avec la réalité/avec les faits lui appartiennent. C'est-à-dire, toutes les conditions de compréhension (toutes les conditions de sens)» [BT 43; variantes dans le texte].

Ainsi, dans le Big Typescript, Wittgenstein pense, d'une part, que certaines choses constituent la vérité et la fausseté (ou la correction et l'incorrection); d'autre part, qu'il y a des conditions qui doivent être remplies pour que des énoncés puissent être comparés à la réalité et jugés vrais ou faux (corrects ou incorrects). De plus, ces conditions qui permettent la comparaison sont les conditions de la compréhension, c.-à-d. que si nous comprenons un énoncé, alors il est pourvu de sens et nous devons être en mesure de décider s'il est vrai ou faux. Les "conditions de la compréhension » sont données par les règles qui constituent la signification, donc par les règles de la «grammaire» qui déterminent le sens des énoncés, et non par des processus psychologiques (nous reviendrons sur le concept de «compréhension » dans la troisième partie). Ainsi, la "grammaire» exprime les conditions structurales qui doivent être remplies pour qu'un énoncé soit pourvu de sens ${ }^{10}$.

L'idée de limite du sens, déjà présente dans le Tractatus, est donc encore très importante pour le Wittgenstein du Big Typescript ${ }^{11}$. Ce n'est pas à la logique et à la forme générale des propositions que Wittgenstein en appelle afin de limiter ce qui peut être dit avec sens (comme dans le Tractatus), mais

à donner les règles pour la combinaison des symboles, c.-à-d. à fixer quelles combinaisons sont pourvues de sens et quelles ne le sont pas, quelles sont permises et quelles ne le sont pas" (Wittgenstein's Lectures Cambridge 1930-1932 (dorénavant WCL30-32), p. 46-7). Voir également p. 66: «La philosophie décrit ce que l'on peut dire avec sens et ce que l'on ne peut pas.» Voir également les p. 48, 49, et 87 .

10. Je traduis Satz par énoncé, autant que possible, et non pas par proposition, parce que dans le Big Typescript Wittgenstein parle constamment de divers genres d'énoncés (des ordres, des questions, des énoncés qui expriment une attente, etc.). Les propositions ne sont plus le seul souci de Wittgenstein.

11. Voir la préface du Tractatus: "Ainsi, l'objectif de ce livre est de tracer une limite à la pensée, ou plutôt - pas à la pensée, mais à l'expression des pensées [...]. Ce sera par conséquent seulement dans le langage que la limite pourra être tracée, et ce qui se situe de l'autre côté de la limite sera simplement du non-sens. ». C'est un trait commun des interprètes "irrésolus » et des "résolus » (irresolute et resolute readers) que de ne pas prendre suffisamment au sérieux l'idée des limites du sens. La lecture "irrésolue» de Hacker, par exemple, attribue au Tractatus l'idée de non-sens profond (voir Hacker I997 et I999). D'autre part, la lecture «résolue» de Diamond, qui reproche à Hacker de ne pas prendre la préface et la fin du livre suffisamment au sérieux (Diamond, I996), voit dans l'idée même des limites du sens un des barreaux de l'échelle qui doit être jetée (voir Diamond 2004 et 2006). Il me semble que les deux lectures du Tractatus comprennent mal la visée de Wittgenstein à cet égard: les lecteurs "irrésolus » ont tendance à oublier que "ce qui se situe de l'autre côté de la limite sera simplement du non-sens» et les lecteurs "résolus » ont tendance à ne pas prendre au sérieux l'idée des limites du sens inscrite dans la préface (dans l'" armature du livre», comme dit Diamond). 
à la "grammaire» et à ses règles. Cependant, tant les règles de la "grammaire » du Big Typescript que la forme générale des propositions du Tractatus ont la même visée fondamentale de déterminer ce qui peut être compté comme proposition pourvue de sens: «Ce qu'est une proposition est déterminé par la grammaire. C'est-à-dire, à l'intérieur de la grammaire. (C'est également ce que visait ma "forme générale de la proposition".)» (BT 77).

Plus tard (dans la deuxième partie) je reviendrai sur l'idée que c'est $\grave{a}$ l'intérieur de la "grammaire» que la nature des propositions est déterminée. Dans le Big Typescript, le but n'est pas d'établir une règle unique qui déterminerait ce qui peut compter comme une proposition, parce que, comme Wittgenstein en est venu à penser, le concept de "proposition" n'a pas de frontières nettes données indépendamment d'un certain système de grammaire (BT 60). D'autre part, ce concept de "proposition » a bien ses règles: mais des règles relatives à un système et qui constituent sa "grammaire déterminée " (BT 62). Il est frappant que les règles de la "grammaire » dans le Big Typescript partagent avec les règles tautologiques du langage dans le Tractatus la caractéristique de "ne rien dire ": "Quand on ajoute la règle à la proposition, le sens de la proposition ne change pas» (BT 24I). Dans ce passage, Wittgenstein a à l'esprit la règle « I $\mathrm{m}$ = la longueur du mètre étalon de Paris" (BT 24I). De même que pour les tautologies dans le Tractatus, "nous pourrions imaginer la règle unie à chaque proposition ${ }^{12}$ " (BT 24I). Ainsi, quand Wittgenstein parle de la «description » des règles de la "grammaire ", il n'a pas proprement en vue une description (propositionnelle), mais plutôt une présentation des règles du calcul qui ont le statut de règles d'un jeu (voir le BT 245).

La différence la plus significative entre le calcul dans le Tractatus et sa nouvelle version dans le Big Typescript est que là, Wittgenstein avait souscrit à une version forte de l'idée de la nature des règles du langage. Dans le Tractatus, toutes les règles fondamentales qui constituent la structure du langage (celle qui exprime toutes les relations logiques) sont réductibles aux règles données par Wittgenstein dans sa notation conceptuelle de la logique vérifonctionnelle - des règles qui sont antérieures à toute expérience possible. Autour de I929-I930, tout juste après être revenu à la philosophie, Wittgenstein découvre que cette version de l'idée du langage comme étant un système de règles fixes, comme un calcul, est erronée. L'idée du langage comme étant un système des règles fixes (un calcul) a survécu cependant, et a été reformulée dans le Big Typescript sous la forme d'une "grammaire» qui doit être découverte dans les calculs effectifs du langage réel: La "philo-

12. Cela est un peu étrange. Joindre la règle « $\mathrm{I} \mathrm{m}=$ la longueur du mètre standard à Paris » à "Wagner a habité à Dresde » semble absurde, et il est difficile d'imaginer une situation dans laquelle il serait approprié de faire une telle conjonction. Wittgenstein pense cependant que la règle ne change pas le sens d'un énoncé quand elle est unie dans des contextes spécifiques (par exemple: les légendes d'une carte, ou la mesure et la définition de l'unité de mesure). 
sophie est concernée par les langages réels et ne doit pas feindre de traiter d'un langage abstrait» (72).

Une conséquence de cette vue est que nous devons accepter que certains concepts (par exemple, «plante» et «ovoïde») n'ont pas de frontières nettes (BT 25 I-2). Nous devons accepter également que certaines régions du langage réel peuvent ne pas avoir les règles que nous cherchons: "Étudions le langage en ce qui concerne ses règles. Si ici et là il n'a aucune règle, alors cela est le résultat de notre recherche » (BT 254). La tâche philosophique en ce qui a trait au langage réel, effectif est, comme dans le Tractatus, celle de le nettoyer (BT 67). Le philosophe grammairien découvre et dresse la «liste des règles» du langage afin de dissiper des confusions philosophiques ${ }^{13}$.

Une autre conséquence de la nouvelle vue est que toutes les règles du langage, et non seulement certaines d'entre elles, sont prises pour des conventions arbitraires dans le sens où elles auraient pu être différentes. Dans le Tractatus, Wittgenstein a pensé que certaines règles étaient arbitraires, à savoir, les règles qui lient le signe et la signification (l'objet), et que d'autres règles, les règles a priori de la logique, ne l'étaient pas parce qu'elles étaient données par l'essence (5.47 I) ou la "nature de la proposition ». Cela est exprimé, tout d'abord, par l'idée que les propositions ont « un prototype logique» :

Si nous transformons en variable une partie constituante d'une proposition, il existe alors une classe de propositions qui sont toutes les valeurs de la proposition variable ainsi créée. En général, cette classe dépend encore de ce que nous donnons comme significations, suivant nos conventions arbitraires, aux parties de la proposition originale. Mais si nous transformons en variables tous les signes dont la signification a été déterminée arbitrairement nous obtenons à nouveau une classe de ce type. Celle-ci n'est cependant plus dépendante d'aucune convention, mais seulement de la nature de la proposition. Elle correspond à une forme logique - un prototype logique. [3.3 I 5 ; c'est moi qui souligne].

Le "prototype logique» peut être exprimé par des variables réelles $(\varphi x)$, de sorte que le $\varphi$ est n'importe quelle fonction n-aire et $x$ ses $n$ arguments. Un tel prototype, une telle forme, est donné a priori, indépendamment de toute expérience:

Nous représentons la chose, la relation, la propriété au moyen de variables, montrant ainsi que nous ne dérivons pas ces idées de cas déterminés qui se présenteraient à nous, mais que nous les possédons en quelque manière $a$ priori [Notebooks, p. 65 ; trad. fr., p. I28].

13. Dans la troisième partie de cet article, nous verrons comment Wittgenstein prétendait nettoyer le langage. Pour ce qui concerne la tabulation des règles et de la liste de règles du langage, voir BT: 68, 7I, I 53, I 55, I78, I88, 245, 250, 253, 426, 548, 758. 
Le prototype, la forme, est commun aux propositions ordinaires non quantifiées et aux propositions élémentaires, mais il n'est cependant pas suffisant pour exprimer leur unité. Naturellement, il n'est pas non plus à même d'exprimer l'unité du langage, c.-à-d., la construction de n'importe quelle proposition, parce que ni la quantification ni les constantes logiques n'y sont immédiatement visibles. Afin d'exprimer cette unité, nous avons besoin d'exemples élémentaires du prototype logique et aussi d'une opération. Selon le Tractatus, une fois que nous avons cela, nous avons la forme générale des propositions et, par là, une manière de produire toutes les formes complexes de propositions possibles. Encore une fois, c'est un signe primitif, une variable (4.53), présent dans la "nature même» des propositions, qui donne l'unité du langage: «On pourrait dire que la seule constante logique est ce que toutes les propositions, par leur nature même, ont en commun les unes avec les autres" (5.47).

La présentation de la forme générale des propositions présuppose que le prototype logique détermine les formes possibles des propositions élémentaires; la forme générale montre, à son tour, que l'opération $N$ peut produire toutes les formes complexes (6). Par là, selon le Tractatus, nous avons tous les éléments essentiels de toute proposition. Notez que rien ici n'est arbitraire, puisque le prototype logique et l'opération $N$ sont donnés $a$ priori. D'une façon ou d'une autre, tout locuteur compétent les connaît implicitement, car c'est cette connaissance implicite qui explique qu'il n'y a aucune proposition dont la forme ne puisse être prévue ou construite (Tractatus 4.5 ).

Une règle qui n'est pas donnée dans la «nature même» des propositions, mais cependant détermine la comparaison de la proposition avec la réalité, est alors une convention arbitraire ou, comme Wittgenstein le dit aussi dans le Tractatus, "une détermination arbitraire ( 5.473). Les conventions arbitraires sont censées présenter les règles qui donnent le contenu ultime de toutes les propositions (par opposition à la forme, qui est donnée par les règles vérifonctionnelles dérivables de la forme générale des propositions). Les conventions arbitraires, les « conventions tacites» - selon l'avis du Wittgenstein du Tractatus — «sont extrêmement compliquées» (4.002). Ces conventions qui pourraient être révélées par l'analyse nous montreraient les noms simples dans le «calcul avec des indéfinissables»(MS. I I I, 3 I). Puisque la visée fondamentale du Tractatus était de présenter la structure $a$ priori du langage au moyen d'une notation conceptuelle, ces conventions compliquées ne sont pas le sujet du livre ${ }^{14}$.

14. La notation conceptuelle du Tractatus inclut au moins les tables de vérité, la forme générale des propositions, les propositions élémentaires logiquement indépendantes, la construction de la quantification (une construction qui produit des composés vérifonctionnels), et les conventions qui éliminent le signe d'identité (voir, respectivement, 5.IOI, 5.5-5.5262, $5 \cdot 53)$. 
En I929, quand il revient à la philosophie, Wittgenstein a pensé qu'il lui fallait les découvrir afin d'expliquer des relations nécessaires entre propositions dont à première vue la notation conceptuelle du Tractatus ne semble pas rendre compte. Cependant, l'analyse que Wittgenstein a amorcée en 1929 (et qu'il a abandonnée tout juste après) n'a pas réussi à dégager ces conventions compliquées qui relient le langage à la réalité et, plus important encore, elle a échoué à prouver que toutes les relations logiques ont un fondement vérifonctionnel. Puisque les règles vérifonctionnelles de la logique sont insuffisantes pour traiter de toutes les relations logiques, l'idée du caractère absolument a priori de la logique et l'idée même d'une structure du langage donnée indépendamment de toute expérience sont peu à peu abandonnées ${ }^{15}$. S'il n'est pas vrai que toutes les relations logiques puissent être exprimées par des règles qui ont une nature vérifonctionnelle, alors il n'y a aucune structure purement a priori du langage. Cela parce que la possibilité des contre-exemples (des relations logiques qui ne peuvent pas être exprimées par renvoi à la structure des propositions) devrait être éliminée en principe par la structure même, a priori, des propositions (Tractatus 5.557). Du même coup, il n'y a plus aucun critère pour établir la distinction entre les règles qui sont données par l'essence des propositions et les règles qui ne sont que des conventions arbitraires. Le Tractatus s'est fourvoyé, selon le Wittgenstein du Big Typescript, au moment où il a supposé que le «langage semble essentiellement être quelque chose à quoi l'on donne une structure pour ensuite la superposer à la réalité » (BT 54; cité intégralement dans le prochain paragraphe). La structure a priori du langage du Tractatus (son «langage abstrait» [BT 72]), ne s'ajuste pas au langage réel. La vraie structure du langage, selon le Wittgenstein du Big Typescript, doit donc être dégagée à même le langage effectif, réel. De cette façon, la notion tractatuséenne d'a priori se dissout, et la nécessité est dorénavant comprise comme l'expression des règles en général.

Le point fondamental dans le traitement que le Big Typescript réserve aux genres de règles et de conventions est que dans un sens toutes les règles du langage se jouent sur le même plan, à savoir, celui d'une détermination arbitraire dans le langage réel. D'autre part, l'idée tractatuséenne d'une «connexion entre le langage et la réalité » (BT 560) est rejetée. Les règles qui supposément relient la réalité au langage sont des règles dont la fonction est

15. Je dis peu à peu parce qu'après avoir abandonné la recherche d'une explicitation vérifonctionnelle des formes "couleur ", "espace» et "temps» Wittgenstein essaye de leur donner une explicitation phénoménologique, qui s'exprimerait dans un «langage phénoménologique». Un tel langage viendrait compléter la notation logique du Tractatus. Le modèle russellien de l'analyse du Tractatus et sa variante phénoménologique de I929 sont abandonnés cette même année. C'est ce dont témoigne la phrase d'ouverture des Philosophische Bemerkungen: «Une proposition est complètement analysée si sa grammaire est complètement mise au clair: et cela quelle que soit la forme d'expression selon laquelle cette proposition se trouve être dite ou écrite.» 
seulement de prolonger le langage, comme c'est le cas pour n'importe quelle autre règle (BT 560). Toutes les règles du langage font partie du même système de règles, et elles dépendent toutes du système ${ }^{16}$. Il n'y pas de termes indéfinissables dans le langage comme calcul, seulement des termes non définis (MS III, 3I). Les mots doivent appartenir à un système, "car [ils] sont de même valeur s'ils ne sont pas situés dans un système grammatical» (BT I 98), c.-à-d. que sans le système ils ne sont que des signes sur du papier ${ }^{17}$. La relation qui consiste à pointer vers un objet ou à lui attacher une étiquette, nous dit Wittgenstein, "n'est significative qu'en raison du système auquel elle appartient» (BT 290) — cela vaut aussi pour les parties de for-

16. Chaque règle particulière est donc arbitraire parce qu'il n'y a désormais plus aucune règle qui fait partie d'une structure a priori du langage, et parce que les règles liées à la «connexion entre le langage et la réalité " sont des règles qui, comme toutes les autres règles, ne font que prolonger le langage. Cependant, le «caractère arbitraire» de la "grammaire» dans le Big Typescript reçoit aussi une formulation plus forte. Wittgenstein soutient que la «grammaire" comme un tout, en tant que système complet de règles, est tout aussi bien arbitraire (voir le BT 233-240; on trouve cette idée déjà en $\mathrm{PB} \ 4$ et 7 ). Contre Wittgenstein, on pourrait penser que chaque règle particulière est arbitraire, mais que le système comme un tout ne l'est pas (c'est là peut être le point de vue de Quine [1969]). Dans le BT, Wittgenstein pense que toute la "grammaire» est arbitraire puisqu'il n'y a aucune justification à l'usage d'un système particulier de «grammaire». Il n'y a aucune justification à la «grammaire » parce que, selon le BT, il n'y a aucun ensemble de propositions vraies desquelles s'ensuit une "grammaire " particulière et parce que toute justification doit avoir lieu à l'intérieur d'un système grammatical. La conséquence de cette vue est que les raisons de choisir une "grammaire» ne peuvent être que de nature pragmatique. Il est intéressant de noter que ce que Wittgenstein dit est très proche (c'est le moins qu'on puisse dire) des distinctions fondamentales que l'on trouve dans la structure de l'argumentation de Logical Syntax of Language de Carnap (voir, par exemple, p. 4-5). La similitude que j'ai en vue n'est pas limitée à la distinction entre les questions de fait et les questions de vérité, et les multiples comparaisons des règles de la syntaxe avec les règles d'un calcul ou d'un jeu. Le point de similitude, je pense, est plus profond: si ce ne sont que les questions pragmatiques qui sont décisives dans le choix d'une "grammaire" (étant donné qu'il n'y a aucune question de fait au sujet d'un tel choix), la «tolérance» de Carnap dans le choix des "grammaires" doit être admise. S'il ne faut accepter aucune "grammaire" comme vraie, il vaut mieux tolérer différentes "grammaires" et choisir celle qui présente les meilleurs résultats pratiques: «[...] Ce que nous disons possible et ce que nous disons ne l'être pas dépend entièrement de notre grammaire, c.-à-d. de ce qu'elle permet. Mais cela est arbitraire! - Certainement, mais ce n'est pas avec n'importe quelle structure que je peux faire une certaine chose, c.-à-d., ce ne sont pas tous les jeux qui sont utiles [...]» (BT 99).

17. C'est la dépendance par rapport au système qui garantit que nos mots ne peuvent pas être simplement inventés ad hoc: "Quand on emploie une proposition elle doit déjà fonctionner d'une manière quelconque. C'est-à-dire qu'on ne l'emploie pas pour associer un bruit à un fait " (BT 199). Dès lors, si Humpty Dumpty veut inventer de nouveaux mots ou choisir ce qu'il veut dire au moyen de ceux-ci, il doit inventer un système auquel ses mots et significations appartiennent (voir Through the Looking Glass, 229). De plus, il ne peut être compris que s'il établit la correspondance entre le système inventé et notre système. Cependant, Humpty Dumpty ne peut inventer son système que parce qu'il a déjà notre système: "Car je ne communique rien non plus si je ne fais qu'associer des groupes de bruits à des faits aléatoires sur une base $a d$ hoc» (BT I98). 
mules algébriques ${ }^{18}$ (BT 662). Si une telle relation ne faisait pas partie d'un système, son horizon nécessaire, personne ne la comprendrait ${ }^{19}$. D'ailleurs, il peut nous arriver d'oublier (et normalement nous l'oublions) l'évènement de l'explication ostensive dans lequel un mot nous a été présenté. Par conséquent, seule la règle peut justifier l'usage futur du mot (BT I99). Cela prouve que ce qui est important dans l'usage d'un mot n'est pas la connexion (causale) qui en est à l'origine, mais la règle exprimée à l'occasion de notre apprentissage du $\operatorname{mot}^{20}$ (BT I 50 ).

Naturellement, les définitions verbales et les définitions ostensives (ou les explications ostensives) sont des choses différentes, mais pas parce qu'il y a une différence essentielle ou de principe entre les deux: elles sont seulement employées différemment dans le langage réel (BT 56). C'est une idée fausse, selon le Big Typescript, que de penser qu'il y a une telle différence de principe:

Règles de grammaire qui établissent une "connexion entre le langage et la réalité » et celles qui ne le font pas. "J'appelle cette couleur "rouge" » est un cas de la première sorte, par exemple. — « $p=p$ » un cas de la seconde sorte de règles. Mais il y a une idée fausse au sujet de cette différence: elle semble être une différence de principe; et le langage semble essentiellement être quelque chose à quoi l'on donne une structure pour ensuite la superposer à la réalité (BT 54).

S'il n'y a aucune structure a priori, la différence entre les deux règles n'est pas une différence de genre, mais de degré de généralité à l'intérieur d'un même système ${ }^{21}$. Ce que Wittgenstein veut nous montrer est qu'«en

18. Voir BT 662: «Ce qui fait qu'il est possible de calculer est le système auquel la proposition appartient, et cela détermine également les erreurs qui peuvent être faites en calculant.» Voir également BT 636-7: «La proposition (Satz) doit appartenir à un système de propositions, et la preuve à un système de preuves. »

19. Moore, dans ses notes des cours de Wittgenstein de I930-I933, dit que le philosophe a insisté sur le fait que "chaque mot ou symbole significatif doit essentiellement appartenir à un "système", et (métaphoriquement) [...] que la signification d'un mot est sa "place" dans un système "grammatical" " (p. 252).

20. Wittgenstein raisonne comme suit: «Si vous demandez à quelqu'un "Comment savez-vous que les mots de votre description rendent ce que vous voyez? ", il pourrait répondre, par exemple, "Je veux dire ceci par mes mots." Mais qu'est-ce que ce "ceci" s'il n'est pas articulé à son tour, s'il n'est pas déjà du langage? Ainsi, "Je veux dire ceci" n'est pas une réponse du tout. La réponse est une explication de la signification des mots» (BT I90). On trouve la même remarque dans un autre texte: "Pour autant qu'elle nous intéresse, l'explication n'opère pas de loin. Elle ne nous intéresse que dans la mesure où elle est employée dans le calcul» (Diktat fuer Schlick, in The Voices of Wittgenstein, I9; trad. fr., p. Iо)

21. Le plus général n'est pas le résultat d'une généralisation empirique, évidemment; ce qui est plus général est tout simplement ce qui est appliqué à plusieurs parties du calcul du langage. Les règles ne peuvent pas être généralisées, seules les propositions le peuvent (BT 240). Les règles vérifonctionnelles peuvent être plus générales que les règles des propositions sur les couleurs, par exemple (BT, II3-5). Quand Wittgenstein écrit au sujet de ce qui est caractéris- 
donnant une explication ostensive des signes on ne sort pas de la grammaire " (BT 43), car ces explications, de même que toutes les règles du langage, «appartiennent au stock de base des explications qui préparent le calcul» (BT 45). En tant qu'éléments du «stock de base ", elles sont simplement des règles et ne sont pas essentiellement différentes d'autres règles de langage. Toute et n'importe quelle règle ne fait rien de plus que préparer l'application du calcul: «Dans ' ${ }_{\alpha} \mathrm{p} \&\left({ }_{\sim} \mathrm{p}=\mathrm{p}\right)$ ' la deuxième partie ne peut être rien de plus qu'une règle du jeu» (BT I 64).

Ce que la reformulation du Tractatus par Wittgenstein manifeste alors, c'est un changement par rapport à l'importance de l'a priori. La distinction entre les règles et les propositions jouait déjà un rôle central dans le Tractatus, mais la caractérisation de certaines règles comme a priori semblait exprimer la nature même de la logique. Une fois que cette structure $a$ priori se superposerait à la réalité, ses règles à leur tour nous conduiraient aux termes simples, indéfinissables. Dans le Big Typescript, la seule distinction fondamentale est la distinction entre la règle et l'énoncé:

En décrivant une notation on pourrait dire: "Dans ce livre, au lieu de 'p ou q' j'écrirai 'pVq' ", et naturellement c'est là un énoncé complet. Mais ce que je veux appeler une «règle », et qui pourrait être écrit "p ou q. =. pVq", n'en est pas une. Ce que j'appelle une "règle» ne doit rien contenir au sujet d'un moment ou d'un endroit particulier (ni même général) de son application, et ne doit pas se rapporter à des personnes particulières (ou à des personnes en général); elle ne doit servir que comme instrument de représentation (BT 246).

L'idée d'a priori n'apporte aucun élément fondamental à l'explication des relations nécessaires dans le calcul du langage. Ce qui est fondamental, c'est l'idée même que les règles sont les conditions de la représentation (ou des instruments de la représentation) et que, en tant que telles, elles ne sont pas des descriptions temporelles ou spatiales. Étant donné que les règles sont toujours relatives à un système, on pourrait dire que nous avons une relativisation de ce qui compte comme nécessaire.

On peut donc résumer les vues de Wittgenstein sur la «grammaire» et ses règles dans le Big Typescript de la façon suivante: les règles du langage sont comprises comme un calcul dans lequel se détermine ce qui peut être dit avec sens et par là être compris. Les règles du calcul, les règles de la "grammaire» enveloppent des définitions ostensives (ou des explications ostensives). Les règles «grammaticales» doivent être découvertes dans les calculs effectifs du langage réel. Ces règles peuvent être dites relativement nécessaires, car elles rendent possible l'application d'un système de langage.

tique dans les règles vérifonctionnelles il mentionne «la généralité inappelable d'une règle cardinale du jeu» (BT I I3). Cependant, en principe nous pouvons jouer différents jeux. 


\section{Autonomie}

La conception du langage comme calcul de Wittgenstein dans le Big Typescript s'appuie sur l'idée que " tout s'effectue dans le langage " (BT 383; mais aussi, sous la forme de remarques manuscrites, p. 379 et 388 ). Ce qui revient à dire que tout ce qui est grammaticalement important (important pour déterminer ce qui a du sens dans le langage) fonctionne comme un calcul autonome. Le calcul est autonome parce que, tel un jeu, il peut être étudié indépendamment de son application (BT 52). De même que nous pouvons distinguer entre les règles d'un jeu et les actions réelles qui consistent à jouer le jeu, nous pouvons aussi distinguer entre les règles de la grammaire et leur application (pour décrire la réalité, pour exprimer des ordres, etc.). Le calcul du langage montre ce qui rend possible l'application des énoncés et des mots (BT I 43), à savoir, ses règles. Par conséquent, tout ce qui est important dans la connexion entre le calcul et le monde et dans l'explication des concepts mentaux (la connexion entre le langage, le monde, et «le mental») fait partie du calcul et s'effectue à l'intérieur du langage (dans la grammaire $)^{22}$. Je commence par une brève discussion de la connexion entre le langage et le monde, et puis je me pencherai sur les concepts mentaux (partie 3).

Nous avons déjà vu un aspect central de l'autonomie du calcul: les définitions ostensives ne fonctionnent pas comme une connexion entre le langage et la réalité qui s'établirait en dehors du langage. On pourrait penser, cependant, que le système du langage ne peut être convenablement étudié que si nous concevons la pensée comme médiation entre le langage et le monde. En fait, nous dit Wittgenstein, une telle connexion n'est qu'une métaphore, parce que nous ne quittons pas le calcul du langage quand nous l'établissons. La connexion entre la pensée et le monde n'est qu'une étape à l'intérieur du calcul du langage. Afin de le montrer, Wittgenstein caractérise tout d'abord "la pensée » comme étant « essentiellement ce qui est exprimé par un énoncé (Satz)»(BT 222). Ce qui veut dire que les pensées sont articulées, qu'elles ne sont pas de simples images amorphes et inarticulées dans l'esprit (j’y reviendrai dans la troisième partie). De même pour les notions comme «signifier quelque chose " et «comprendre ${ }^{23}$.

22. Le deuxième point a une saveur behavioristique. Wittgenstein en fait clairement état: "L'aspect behavioristique de notre discussion consiste seulement dans le fait que nous ne distinguons pas "l'externe" et "l'interne". Puisque je ne m'occupe pas de la psychologie" (BT 284). Ce que Wittgenstein nous dit n'est pas que nous ne pouvons pas les distinguer. Nous le pouvons; cependant, cette distinction ne soutient pas ce que les philosophes veulent en faire: prendre des processus psychologiques comme de meilleurs symboles que des signes sur du papier (voir le BT 283). J'explique cela dans les prochaines pages. Wittgenstein ne veut pas non plus ramener les concepts psychologiques au seul comportement (voir, par exemple, sa remarque sur l'attente d'une chose dans BT 373; cette remarque est citée ci-dessous).

23. Pour ce qui concerne la compréhension, voir BT I43: "Alors "comprendre un énoncé" est-il du même genre que "maîtriser un calcul" ? Et donc aussi du même genre que: être capable de multiplier? C'est ce que je crois.» Pour ce qui concerne le «signifier quelque 
En second lieu, Wittgenstein exemplifie la connexion entre la pensée et le monde par divers genres d'énoncés, c'est-à-dire, par divers genres d'expressions de pensées: descriptions, énoncés qui expriment une attente (mais aussi des expressions de souhaits, de croyance, etc.) et des ordres. Dans toutes sortes d'énoncés, la relation à la réalité « est toujours une étape dans le calcul» (BT 2 I6). J'expliquerai ce point en prenant comme exemples des énoncés descriptifs et des expressions d'attente.

Si on compare une proposition à la réalité, on peut décrire la réalité et comparer la description avec la première proposition:

Si je veux examiner la réalité pour voir si elle est conforme à une proposition, alors je peux également le faire en la décrivant et en voyant si la même proposition en résulte. Ou je peux - selon les règles grammaticales - traduire la réalité dans le langage de la proposition et puis effectuer la comparaison à l'intérieur du domaine du langage [BT 204].

On ne quitte pas le calcul quand on détermine si une proposition est vraie. Quand on veut établir un accord entre la proposition et la réalité, on a tout simplement deux propositions à l'intérieur du domaine du langage parce que l'on peut décrire la réalité d'abord et ensuite comparer la description à la proposition. On peut le faire parce que la réalité est traduisible, c.-à-d. qu'elle est déjà articulée par des règles de "grammaire » quand nous faisons la comparaison. Nous commençons par un système articulé des règles et nous ne le quittons jamais pour de bon. Nous commençons par le système entier des règles et non pas, par exemple, par des explications ostensives (ou des définitions ostensives). Quand nous utilisons le langage pour décrire quelque chose, nous sommes déjà soumis à ses règles systématiques (BT 200).

Pour vérifier si une attente a été remplie, encore une fois, on ne quitte pas le calcul: "L'attente et l'événement se touchent dans le langage» (BT 37I). Supposez que je m'attende à ce que quelqu'un nommé Pierre arrive. Qu'est-ce qui caractérise «Je m'attends à ce qu'il vienne»? Wittgenstein répond:

Je pourrais sûrement marcher de long en large sans m'attendre à ce qu'il vienne, et je pourrais tout aussi bien regarder ma montre, etc.; de sorte que ce n'est pas cela qui caractérise «m'attendre à ce qu'il vienne ». Ce qui le caractérise n'est fourni que par ces mots mêmes. Et «il» veut dire la même chose que dans l'affirmation «il vient ", et "vient » veut dire la même chose que dans cette affirmation, et leur combinaison ne signifie rien de différent. C'est-à-dire qu'une explication ostensive du mot «il» vaut pour les deux propositions [BT 373].

chose", voir BT 203: "Si le "signifier" [Meinen] doit avoir un poids ou une importance quelconque pour nous, alors un système de significations [Meinungen] doit être coordonné au système de propositions, quel que soit le genre de processus représenté par les significations. » 
Le phénomène du «marcher de long en large » ne caractérise sûrement pas suffisamment l'attente, parce que l'on peut marcher de long en large pour d'autres raisons. Cela ne veut pas dire, cependant, que nous devons faire appel à quelque chose "à l'intérieur de l'esprit " pour caractériser l'attente (je reviendrai sur ce point plus tard). Ce qui caractérise le rapport de l'attente et de son remplissement est que la même explication ostensive peut être employée dans les deux cas, celui de l'attente et celui de la description de son remplissement. Si je dis, avant que Pierre arrive, «je m'attends à ce qu'il vienne» et, après qu'il est arrivé, "il est venu», le pronom dans les deux énoncés se rapporte à une explication ostensive, qui est une règle à l'intérieur du langage (comme nous l'avons déjà vu ci-dessus).

La dérivation du remplissement de l'attente opère, nous dit Wittgenstein, comme la dérivation d'une nouvelle étape dans un calcul:

C'est une étape dans un calcul qui mène d'une attente au remplissement. En effet le calcul

$$
\underline{25 \times .25}
$$

50

$\underline{\mathbf{2 5} 5}$ est lié au résultat 625 exactement de la même manière que l'attente l'est au remplissement. Et dans la mesure - et seulement dans cette mesure - où ce calcul est une image du résultat, l'attente est également une image du remplissement. Et la mesure dans laquelle le résultat est déterminé par le calcul

- voilà la mesure dans laquelle le remplissement est déterminé par l'attente [BT 376].

Dans les deux cas, dans la dérivation mathématique et dans la dérivation du remplissement de l'attente, les dérivations sont faites selon une règle (nous agissons "selon une règle" [BT 378]). Ce qui relie la première étape (l'attente ou $25 \times 25$ ) à la deuxième étape (le remplissement de l'attente ou 625 ) est une règle à l'intérieur du système de la "grammaire». Si l'on attend Pierre, c'est Pierre et non pas Jean ou Paul que l'on attend, de la même manière que le résultat du calcul sera un nombre parmi plusieurs autres contrastants à l'intérieur du système numérique. Dans les deux cas, il y a un résultat qui est correct et d'autres qui sont incorrects (si c'est Jean qui arrive au lieu de Pierre, l'attente n'est naturellement pas remplie). Les règles implicites dans l'expression de l'attente réduisent ainsi le nombre de faits possibles qui la remplissent et déterminent ce que l'on doit attendre (l'«image du remplissement»). De la même manière, les règles de la multiplication déterminent un résultat parmi beaucoup de résultats contrastants possibles.

\section{Les opérations du calcul et le non-sens: un exemple}

L'autonomie du calcul dans le Big Typescript est également censée contrecarrer l'idée que penser, signifier, viser, etc. sont essentiellement des processus cachés dans un milieu mystérieux, l'esprit, conçu comme un mécanisme qui 
injecte la signification dans le langage: «La pensée ne doit pas être comparée à l'activité d'un mécanisme que nous voyons de l'extérieur, mais dont il nous faut encore pénétrer les rouages internes» (BT 22I). «La pensée [nous l'avons déjà vu] est essentiellement ce qui est exprimé par un énoncé (Satz)» (BT 222). Ainsi, comprendre une pensée est comprendre un énoncé (BT I). Si quelqu'un comprend un énoncé, il peut expliquer son sens: " "Comprendre" - je veux dire par là le corrélat d'une explication de sens " (BT II). Toute interprétation des signes est toujours corrélée à une explication à l'intérieur du calcul. On explique le sens d'un énoncé ou on l'interprète en présentant les règles de la grammaire qui y sont utilisées (BT 79). C'est aussi de cette manière que l'on explique ce qui est signifié et l'intention (BT 374 et BT 272-92). Ce qui est signifié est donné par une explication de la signification, qui est un énoncé (BT 2). Cette explication doit contenir implicitement une règle. L'explication ne nous aide pas à trouver la signification (BT 34); elle nous donne la signification elle-même (laquelle est constituée par les règles qui permettent de situer un mot à l'intérieur de la «grammaire»).

Ainsi, penser, signifier, viser et comprendre sont autant d'opérations corrélées à l'intérieur d'un calcul, des activités qui peuvent être rendues sous la forme d'une explication, c.-à-d. d'un énoncé avec une grammaire. Se demander ce qui gît derrière ou bien sous ces activités revient nécessairement à poser une question au sujet de leur position à l'intérieur du calcul (dans le système): "Si l'on demande les raisons derrière un acte individuel de pensée (un acte de calcul), la réponse que l'on obtient est une analyse d'un système auquel l'acte appartient» (BT 23I).

Si l'on pense ou signifie quelque chose, on pense ou signifie quelque chose par opposition à d'autres possibilités à l'intérieur du système du langage (BT 290, 280). Si l'on demande à quelqu'un d'apporter une fleur jaune, par exemple, alors on demande une fleur par opposition, disons, à une tasse de thé, et une fleur jaune par opposition à une rouge. Ainsi, ce que l'on vise ou signifie est déterminé à l'intérieur d'un système de possibilités. Si ce qui est signifié doit recevoir une spécification supplémentaire, alors ce que l'on reçoit, c'est une explication contrastive (un énoncé) à l'intérieur du système. Le fondement d'une explication atteint sa limite dans les règles de la grammaire, les points fixes du langage, qui ne sont pas ambigus et, en tant que tels, règlent toutes les disputes: «Les seules choses qui sont exactes et non ambiguës et indisputables sont les règles grammaticales, qui au bout du compte doivent montrer ce qui est signifié » (BT 374).

Comme des parties d'équations, les explications introduisent certaines transformations qui peuvent prendre la place de ce qui est expliqué. Plusieurs de ces substitutions peuvent être introduites en accord avec le système de règles auquel elles appartiennent. Quand elles semblent être insuffisantes, par exemple si des disputes arrivent, la dernière instance d'appel est les règles mêmes qui composent le système. C'est-à-dire, les justifications don- 
nées aux démarches spécifiques entreprises dans le langage sont complètes quand les règles sont données, c'est dans les règles que les explications touchent à leur fin:

Si on me demande: "Pourquoi avez-vous écrit $5^{2}$ ?" et que je lui réponds: "Il faut pourtant que j'élève au carré ", alors c'est une justification - et une justification complète. Demander une justification au sens dans lequel celle-ci n'en est pas une est dépourvu de sens [BT 279].

Les philosophes, selon le Wittgenstein du Big Typescript, essaient cependant d'aller "plus profondément ", pour ainsi dire, et posent des questions au sujet de la signification qui vont apparemment au-delà du système ou du calcul avec des règles fixes qui donnent d'abord la structure nécessaire à ce qu'une question soit posée ${ }^{24}$. Les questions qui semblent aller au-delà du système sont de cette sorte: "Comment pouvez-vous savoir ce que quelqu'un veut vraiment dire si vous n'avez accès qu'aux signes parlés? » ou "Comment pouvons-nous être sûrs que nous voulons dire la même chose par "jaune" ?" Il est très utile de comparer un tel non-sens à ce que Wittgenstein dit au sujet de certaines combinaisons algébriques:

Ce qui rend le calcul possible est le système auquel il appartient; et cela détermine également les erreurs qui peuvent être faites dans le calcul. Par exemple, $(a+b)^{2}$ est égal à $a^{2}+2 a b+b^{2}$, et non pas à $a^{2}+a b+b^{2}$; mais $(a+b)^{2}=-4$ n'est pas une erreur de calcul possible dans ce système [BT 662].

Pour le Wittgenstein du Big Typescript (mais également pour celui du Tractatus $^{25}$ ), les philosophes quittent le système de règles sans s'en rendre compte quand ils décident d'étudier la nature des entités mentales: « $(a+b)^{2}$ $=-4$ » ressemble à " $\mathrm{a}^{2}+2 \mathrm{ab}+\mathrm{b}^{2}$ ", et cependant aucune signification n'a été donnée à la première expression. C'est-à-dire qu'il n'y a pas de règles (pas de système) pour la première «équation ». De la même manière, la question "Comment pouvez-vous savoir ce qu'il veut dire si vous n'avez accès qu'aux signes parlés?" ressemble à "Comment pouvez-vous savoir s'il est à la maison si la porte est fermée? ». On peut répondre à la dernière question par une des nombreuses réponses possibles à l'intérieur du système (par exemple, "J'ai entendu un bruit" "Il m'a appelé et m'a dit qu'il serait à la maison », etc.). Cependant, il n'y a aucune réponse dans notre système qui puisse être une réponse pour le premier genre de question (ce n'est pas une erreur de calcul), à moins que l'on trivialise et la question et la réponse: "Je sais ce qu'il veut dire parce que les signes parlés expriment ce qu'il veut dire.»

Pour le Wittgenstein du Big Typescript, les questions philosophiques au sujet de la signification, de l'intention, etc., semblent supposer qu'il doit

24. La limite des raisons est donnée par le système dans lequel des raisons sont données: "(Une raison ne peut être donnée que dans un jeu.) La chaîne de raisons touche à une fin — sa fin dans ce jeu» (BT I 88).

25. On ne doit pas oublier que dans le Tractatus «der Gedanke ist der sinvolle Satz» (4). 
y avoir un mécanisme d'injection de signification caché dans le «mental $»^{26}$. Des phrases parlées ou écrites sont prises comme étant la surface, comme si les mots pouvaient tout juste insinuer la signification sans pouvoir l'exprimer. Mais qu'y a-t-il dans le mental qui n'est pas dans le système ou calcul du langage? Pour nous empêcher d'essayer de quitter le système de règles et donc pour éviter une pseudo-recherche sur les entités mentales, Wittgenstein raisonne en deux étapes. D'abord, si des entités mentales (ou des états mentaux) sont articulées, elles correspondent à un énoncé:

La compréhension est corrélée à l'explication; et dans la mesure où elle ne le fait pas, elle est inarticulée et donc sans intérêt pour nous; ou alors elle est articulée et corrélée à l'énoncé lui-même, dont nous voulions décrire la compréhension [BT II].

La compréhension articulée est exprimée par une explication, qui est elle-même articulée (c'est un énoncé). Ainsi, nous sommes revenus au calcul, à la "grammaire». En second lieu, si des entités mentales ne sont pas articulées, elles sont inutiles parce que superflues dans toute explication de ce qui est signifié:

Toutes mes réflexions vont toujours dans le sens de prouver qu'il est inutile de concevoir la pensée comme une hallucination. En d'autres termes, cela est superflu et ne touche pas au problème. Car ni même l'hallucination - aucune image - ne peut franchir le fossé entre l'image et la réalité, et à cet égard aucune image n'est meilleure que les autres [BT 292].

Cela signifie qu' "un processus psychologique ne peut pas faire plus que des signes écrits sur du papier» (BT 283). Pourquoi pas? Eh bien, parce que les processus psychologiques ou les entités mentales ne peuvent franchir le fossé entre le langage, la pensée et la réalité que dans la mesure où les mots le peuvent aussi. Supposez que quelqu'un veuille trouver, qu'il cherche, etc., un objet X et qu'il ait des images à l'esprit quand il cherche ou trouve le X. (Wittgenstein n'a naturellement pas besoin de nier qu'on a des images ou des «hallucinations", comme il le dit également, dans de pareilles circonstances.) La question importante est le rôle que l'image (l'entité mentale) joue dans cette tâche. Tout d'abord, une image n'est pas une condition nécessaire pour que quelqu'un cherche le X. Et même si parfois on a une image de $\mathrm{X}$ tout en cherchant $X$, on peut parfaitement le faire sans image du tout (ce qui en fait arrive la plupart des fois). En second lieu, l'image ne peut pas justifier quelqu'un qui choisit ou ne réussit pas à choisir le $\mathrm{X}$ en question. Supposez que vous disiez «S'il vous plaît, Jean, apportez-moi une fleur jaune.» Il

26. Ici, comme dans beaucoup d'autres textes de Wittgenstein de la période intermédiaire, il peut sembler «naturel» de penser que les remarques sur l'intention, sur le signifier et les autres concepts mentaux constituent en quelque sorte une critique du Tractatus; voir, par exemple, E. Ammereller (200I) et P.M.S. Hacker (I997). À mon avis, il ne s'agit pas de cela; je ne peux m'en occuper ici, mais voyez Engelmann (20I 2a et 20I2b). 
serait absurde que Jean revienne avec une fleur rouge (ou une tasse jaune) en disant que l'objet qu'il vous a apporté correspond exactement à son imageguide mentale. Vous diriez certainement à Jean, de façon plus ou moins polie, qu'il devrait vous apporter une fleur jaune et non pas quelque chose qui ressemble à ses images mentales. Les entités mentales sont ainsi totalement dénuées de pertinence dans la justification de ce qui est signifié ou visé (BT 372). Troisièmement, les images mentales par elles-mêmes ne peuvent pas effectuer le travail qu'elles sont censées accomplir. Si elles avaient une utilité quelconque, elles détermineraient la signification des mots. Mais comment serait-il possible qu'elles la déterminent? On pourrait penser que le signe propositionnel est ambigu et peut être compris de plusieurs manières. On pourrait également supposer que les entités mentales, les images, déterminent ou fixent la signification des signes. Cependant, pourquoi les images devraient-elles faire mieux que les mots et leurs règles (ou même faire mieux que les images physiques) ? De la même manière qu'on peut mal comprendre un mot, une image peut être mal comprise; une image peut être aussi ambiguë qu'un mot. Car une image ne nous dit pas par elle-même de qui ou de quoi elle est une image. L'appel aux similitudes entre l'image et l'objet est tout aussi bien insuffisant pour s'acquitter de cette tâche. Comme n'importe quel autre dispositif que l'on pourrait utiliser pour l'identification du remplissement de l'attente, de ce qui satisfait un ordre, etc., l'utilisation d'une image mentale comme guide présuppose que je comprends ce que je dois faire de cette image:

Il n'est évidemment nullement inconcevable qu'en cherchant une fleur jaune une personne puisse employer une image mentale, tout comme une autre pourrait employer un échantillon de couleur, ou une troisième personne pourrait d'une manière quelconque - employer l'image d'une réaction qui serait déclenchée par la chose qu'il cherche (une sonnette).

Mais peu importe ce qu'elle emploie dans sa recherche (peu importe quel est le paradigme), rien ne la force à reconnaître [anerkennen] ce qu'à la fin elle reconnaît effectivement comme la chose qu'elle cherchait; et, ici encore, la justification qu'elle fournit alors pour le résultat, au moyen de mots ou d'autres signes, ne justifie cette chose que par rapport à une description différente dans le même langage [BT 37I-2].

Ainsi, «le langage différent» des images mentales ne fait qu'ajourner le problème de l'identification (en admettant que ce soit un problème), car rien n'oblige quelqu'un à prendre l'objet pour ce qui est représenté dans l'image. Et, encore une fois, la justification que l'on donne est simplement une explication à l'intérieur d'un système; c'est une explication qui exclut d'autres possibilités données par le système. Ainsi, une image mentale ne peut pas venir à bout du travail qu'elle est censée accomplir. La tentation est proche de supposer des images gouvernées par des règles, c.-à-d., des images qui nous donnent les règles de l'usage des mots. Mais, dans ce cas-là, nous pouvons garder les règles et nous débarrasser des images. 
La conception du langage comme calcul met donc en œuvre une double stratégie dans le Big Typescript: elle vise à éviter la psychologisation de la "grammaire» (en mettant en corrélation les divers concepts psychologiques à l'intérieur d'un système), mais elle vise aussi à nous détourner d'une recherche pseudo-scientifique (philosophique) sur le rapport entre le langage et la réalité. C'est à l'intérieur du calcul du langage que la réalité et le langage "entrent en connexion". Si nous éclaircissons la grammaire de "comprendre", nous élucidons également la grammaire de «viser» et de "signifier». Par là, nous pouvons exclure certaines questions comme étant dépourvues de sens, à savoir, les questions qui ne respectent pas le système de règles fixes de la "grammaire». Si la philosophie n'est pas une science de la nature, elle n'étudie pas le cerveau et son rapport avec l'environnement (la réalité); si la philosophie n'est pas de la psychologie, elle n'étudie pas non plus les opérations mentales. C'est ainsi le langage qui est le sujet de la philosophie. La compréhension des opérations du calcul autonome du langage exprime donc l'autonomie de la philosophie. C'est pourquoi les remarques rassemblées dans le Big Typescript indiquent une conception philosophique fermée du langage fondée sur l'idée du langage comme calcul.

La conception du langage comme calcul dans le Big Typescript doit être vue comme une reformulation (une nouvelle version) du calcul du Tractatus, mais tout aussi bien comme une conception philosophique encore éloignée des derniers écrits de Wittgenstein. Le lecteur attentif des Recherches philosophiques doit comprendre que Wittgenstein y critique non seulement la philosophie du Tractatus, mais également celle qui était la sienne à l'époque du Big Typescript. C'est bien ce qui semble ressortir du passage où il admet qu'il fut captif de l'idée que "si on énonce une phrase et si on veut la dire, ou si on la comprend, on opère un calcul selon des règles définies » $(\mathbb{S}$ I). Puisque dans le Big Typescript les notions de "signification", de "compréhension" se trouvent coordonnées dans un calcul, il semble évident que cette œuvre est la cible (ou au moins une des cibles) de Wittgenstein dans le $\mathbb{8}$ I des Recherches philosophiques. Par conséquent, on ne doit pas penser que les deux œuvres expriment la même conception de la "grammaire ${ }^{27}$. Cependant, la manière dont la philosophie de Wittgenstein se développe et la raison pour laquelle elle se développe après le Big Typescript est au-delà de la portée de cet article ${ }^{28}$.

Traduit de l'anglais par Bento Prado Neto

27. Hacker croit que les deux œuvres partagent essentiellement la même conception de la "grammaire», car il pense que, dans les Recherches philosophiques, Wittgenstein défend une sorte de conception de dicto de la nécessité (voir Baker et Hacker 2005, chapitre XIII; Hacker 2000, chapitre III).

28. Je discute son interprétation longuement dans Englemann 20 I I. 


\section{Bibliographie}

Ammereller, E. «Wittgenstein on Intentionality ». In Wittgenstein: A Critical Reader, H.-J. Glock (ed.), Oxford, Blackwell Publishers, 200I, 59-93.

Baker, G. The Voices of Wittgenstein (The Vienna Circle). Ludwig Wittgenstein and Friedrich Waismann. Original German Texts and English translations. Transcription, introduction by Gordon Baker (ed.), Routledge, 2003 ; trad. fr. Dictées de Wittgenstein à Waismann et pour Schlick, vol. I, PUF, I 997.

Baker, G. \& P.M.S. Hacker. Wittgenstein: Meaning and Understanding (Part I Essays). Vol. I of an analytical commentary to the Philosophical Investigations. Second, extensively revised edition, Oxford, 2005.

Carnap, R. The Logical Syntax of Language. A. Smeaton (trad.), Paterson, New Jersey, Littlefield, Adams \& Co., I959.

Carroll, L. Alice in Wonderland and Through the Looking Glass (Grosset \& Dunlap, I983).

Diamond, C. The Realistic Spirit: Wittgenstein, Philosophy and the Mind, Cambridge, Mass., The MIT Press, I996.

—. "Peter Winch on the Tractatus and the Unity of Wittgenstein's Philosophy». In Wittgenstein: The Philosopher and His Work. Publications of the Austrian Ludwig Wittgenstein Society, Volume 2. A. Pichler and S. Saatela (eds.), Ontos Verlag, 2006, I4I-I7I.

- "Criss-cross Philosophy". In Wittgenstein at Work. Method in the Philosophical Investigations. E. Ammereller and E. Fischer (eds.), Routledge, 2004, 2OI-220.

Engelmann, M. L. Wittgenstein's Philosophical Development (Phenomenology, Grammar, Method, and the Anthropological Perspective). À paraître, Macmillan Palgrave, 20I 2a.

—. "Wittgenstein's New Method and Russell's "The Analysis of Mind” ". À paraître, Journal of Philosophical Research, 2012b.

—. "Wittgenstein's "Most Fruitful Ideas" and Sraffa ». À paraître, Philosophical Investigations, 20I2c.

—. "What Wittgenstein "Grammar" Is Not» (On Garver, Baker and Hacker, and Hacker on Wittgenstein on "Grammar"). Wittgenstein-Studien 2, 2OI I, 7I-IO2.

Hacker, P. M. S. "Naming, Thinking and Meaning in the Tractatus", Philosophical Investigations, I999, I I9-I35.

- Insight and Illusion, 2 ed. Bristol, Thoemes Press, I997.

- Wittgenstein: Mind and Will (Part I Essays). Vol. 4 of an analytical commentary on the Philosophical Investigations, Oxford, 2000.

Kienzler, W. Die Stellung des Big Typescripts in Wittgensteins Werkentwicklung. In Wittgensteins, grosse Maschinenschrift' (Untersuchungen zum philosophischen Ort des Big Typescripts (TS 213) im Werk Ludwig Wittgensteins), S. Majestschack (ed.), Peter Lang, 2006, I I-30.

Moore, G. E. «Wittgenstein's Lectures 30-33». In Philosophical Papers, New York, Collier Books, I966, 252-325.

Quine, W. V. Ontological Relativity and Other Essays, New York, Columbia University Press, I969.

Von Wright, G. H. Wittgenstein, Minneapolis, University of Minnesota Press, I980. Wittgenstein, L. Tractatus Logico-philosophicus. B. F. McGuiness et D. Pears (trad.), Routledge, 2004 . 
-. Philosophical Investigations. Édition allemand-anglais. A. Anscombe (trad.), Blackwell, 200I.

- Wittgenstein's Nachlass. The Bergen Electronic Edition, Oxford, 2000.

—. The Big Typescript. M. Nedo (ed.), Zweitausendeins, 2000.

- The Big Typescript: TS 213. German-English Scholars' Edition. C.G. Luckardt, and A.E. Aue (eds. and transl.), Blackwell, 2005.

- Notebooks 1914-1916. G.E.M. Anscombe (transl. and ed.) and G.H. Von Wright, (ed.), Chicago, University of Chicago Press, I979; trad. fr., Carnets 1914-1916, Paris, Gallimard, I97I.

—. Philosophische Bemerkungen. Werkausgabe Band 2, Suhrkamp, 1989.

—. Wittgenstein und der Wiener Kreis. Werkausgabe Band 3, Suhrkamp, 1993.

—. The Blue and the Brown Books. Harper Torchbooks, 1960.

—. Philosophische Grammatik. Werkausgabe Band 4, Suhrkamp, I989; trad. fr., Grammaire philosophique, Gallimard, 1980.

—. Wittgenstein's Lectures Cambridge, 1930-1932. From the notes of John King and Desmond Lee (ed.). University of Chicago Press, I989. 